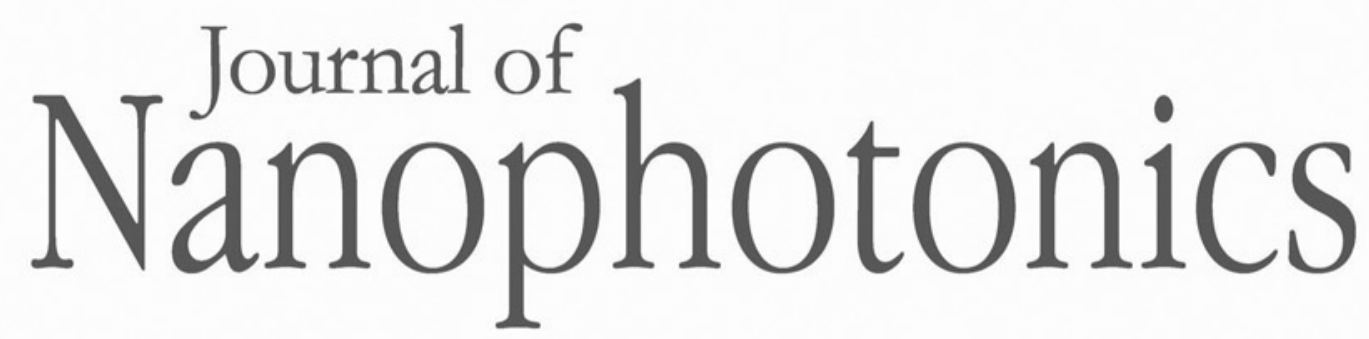

\title{
Photonic crystal fiber-based plasmonic biosensor with external sensing approach
}

\author{
Ahmmed A. Rifat \\ Md. Rabiul Hasan \\ Rajib Ahmed \\ Haider Butt
}




\title{
Photonic crystal fiber-based plasmonic biosensor with external sensing approach
}

\author{
Ahmmed A. Rifat, ${ }^{\mathrm{a}, \mathrm{b}}$, . Md. Rabiul Hasan, ${ }^{\mathrm{c}}$ Rajib Ahmed, ${ }^{\mathrm{d}}$ and \\ Haider Butt ${ }^{d}$ \\ ${ }^{a}$ Australian National University, Nonlinear Physics Centre, Research School of Physics and \\ Engineering, Canberra, Australia \\ ${ }^{b}$ University of Malaya, Integrated Lightwave Research Group, Department of Electrical \\ Engineering, Kuala Lumpur, Malaysia \\ ${ }^{c}$ Rajshahi University of Engineering and Technology, Department of Electronics and \\ Telecommunication Engineering, Rajshahi, Bangladesh \\ ${ }^{\mathrm{d}}$ University of Birmingham, Nanotechnology Laboratory, School of Engineering, Birmingham, \\ United Kingdom
}

\begin{abstract}
We propose a simple photonic crystal fiber (PCF) biosensor based on the surface plasmon resonance effect. The sensing properties are characterized using the finite element method. Chemically stable gold material is deposited on the outer surface of the PCF to realize the practical sensing approach. The performance of the modeled biosensor is investigated in terms of wavelength sensitivity, amplitude sensitivity, sensor resolution, and linearity of the resonant wavelength with the variation of structural parameters. In the sensing range of 1.33 to 1.37 , maximum sensitivities of $4000 \mathrm{~nm} / \mathrm{RIU}$ and $478 \mathrm{RIU}^{-1}$ are achieved with the high sensor resolutions of $2.5 \times 10^{-5}$ and $2.1 \times 10^{-5}$ RIU using wavelength and amplitude interrogation methods, respectively. The designed biosensor will reduce fabrication complexity due to its simple and realistic hexagonal lattice structure. It is anticipated that the proposed biosensor may find possible applications for unknown biological and biochemical analyte detections with a high degree of accuracy. (C) 2017 Society of Photo-Optical Instrumentation Engineers (SPIE) [DOI: 10 1117/11]NP12.012503
\end{abstract}

Keywords: surface plasmon resonance; photonic crystal fiber; fiber optic sensor; biosensor; sensitivity; resolution.

Paper 17034SS received Mar. 24, 2017; accepted for publication Jun. 5, 2017; published online Jun. 19, 2017; corrected Aug. 17, 2017.

\section{Introduction}

Over the last decades, photonic biosensors have attracted intense attention due to their promising applications in several fields including medical diagnostics, biomolecules detection, biochemicals detection, environment monitoring, etc. ${ }^{\mathrm{B}}$ S Several sensing approaches such as fiber Bragg grating, microring resonator, resonant mirror, multimode interference, and surface plasmon resonance (SPR) have been widely explored. ${ }^{\mathbb{B}}$ Among them, SPR-based sensors have gained maximum interest as they offer high sensitivity and better detection accuracy of unknown analytes. The earlier SPR biosensors were mostly based on the Kretschmann set-up configuration, where a prism and active metal layer were used. Since prism-coupling SPR sensors are bulky and consist of moving optical and mechanical parts, they are not suitable for remote-sensing applications. ${ }^{-T}$ The use of fiber optic can greatly reduce the major challenges of prism-based biosensors. Photonic crystal fiber (PCF) is more convenient compared with the regular fiber in SPR sensing. In PCF-based SPR sensing, an evanescent field is the focal parameter to enhance the sensor performance. Conventional optical fiber consists of core and cladding having different materials. The index contrast between core and cladding is relatively smaller, which limits the

*Address all correspondence to: Ahmmed A. Rifat, E-mail: Eifatahmmed@osamember org 
efficient control of the evanescent field. However, PCFs offer wider design space by taking advantage of large index contrast between core and cladding. By varying the structural parameters such as air-hole diameter, pitch, and crystal arrangement, the evanescent field can be easily tailored. As a result, PCFs have been widely used instead of conventional optical fiber ${ }^{\text {B⿴囗 }}$ In PCFs, SPR phenomena can be easily observed, and performance of the sensor can be controlled by varying the fiber's design parameters (air-hole radius, pitch size, etc.). The structures of PCFbased biosensors can be realized using existing fabrication technologies. In addition, PCF-based SPR biosensors allow reduction of sensor size and permit easy manipulation of the guided electromagnetic field. To create the SPR effect, a metal coating is required to be deposited either in the inner air holes of the PCF or on the outer surface of the PCF. Several plasmonic materials including silver, gold, and copper have been widely studied for SPR sensors. ${ }^{\text {a }}$ Although silver material offers a sharp resonance peak, it is chemically unstable and very susceptible to oxidization, which reduces the sensing performance. ${ }^{\mathbb{D}}$ To prevent oxidization, an effective approach is to deposit nanoscale graphene layers on the top of the plasmonic material ${ }^{0}$ However, maintaining a uniform thickness of this additional layer is difficult, and it also increases the overall manufacturing cost. On the other hand, gold is chemically stable and provides a high resonance peak. Unlike copper, gold material does not suffer from oxidization. In PCF SPR biosensor, an evanescent field propagating toward the cladding region strikes the plasmonic metal interface that excites the free electrons. When the frequency of the oscillating surface electrons and incident photons are matched, a sharp loss-peak is observed. Mathematically, SPR phenomenon happens when the real refractive index (RI) $\left(n_{\text {eff }}\right)$ of the guide mode and the real $n_{\text {eff }}$ of the surface plasmon polariton (SPP) mode are equal. Under this condition, maximum mode power transfers from the core-guided mode to the SPP mode.

To date, several distinct PCFs biosensors based on the SPR phenomenon have been proposed Refs. 13-15. In such PCFs, several micron-scale air holes are selectively coated with plasmonic metal as well as selectively infiltrated with liquids. Although these types of biosensors (inside sensing) offer high sensitivity, they are difficult to realize from the fabrication point of view. Maintaining an accurate coating inside the air holes to deposit several metal layers is also a challenging task. To eliminate the shortcomings of these biosensors, externally metal-coated PCF SPR sensors have been introduced. The D-shaped PCF SPR sensors have been reported in Refs. 12, 16, 19, showing the improved sensing performances. Tian et al. reported a D-shaped

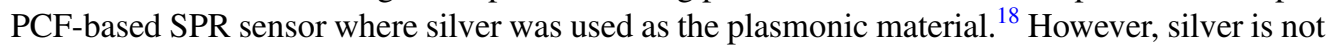
chemically stable and prone to oxidize easily. It achieved the maximum sensor resolution of $7300 \mathrm{~nm} / \mathrm{RIU}$ at analyte RI 1.38. However, such D-shaped PCFs require additional effort to polish the predefined section of the PCF, which increases the fabrication difficulties. To date, several improved PCF SPR sensors have been reported where a plasmonic metal layer is placed

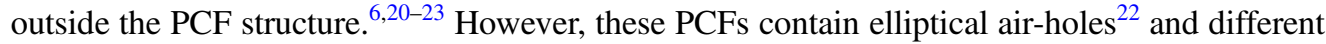

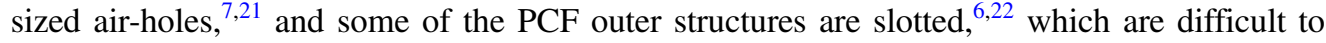
control during fabrication. Additionally, most of the reported external sensor approaches used a small central air hole to facilitate penetration of the evanescent field. Practically, achieving such PCF structures are challenging. Recently, metallic nanowire-based plasmonic sensors have been reported using silver nanowires. 때 Based on the plasmonic resonance, ultrathin metallic nanolayer plasmonic tapered fiber sensors have also been explored in the literature. [6]

In this paper, we propose a simple solid-core PCF-based SPR biosensor. Unlike the existing biosensors, the proposed sensor has minimum irregular design parameters, which reduces the fabrication complexity. Chemically stable gold material is coated on the outer surface of the PCF structure, which is more straightforward for practical fabrication. The proposed biosensor also allows a simple detection approach since unknown analyte can be detected by flowing it through the outer gold surface.

\section{Design and Simulation Methodology}

Figure (a) shows the cross-section of the proposed PCF preform structure during the stacking process. As illustrated in the figure, the central core and four missing air holes in the second ring 


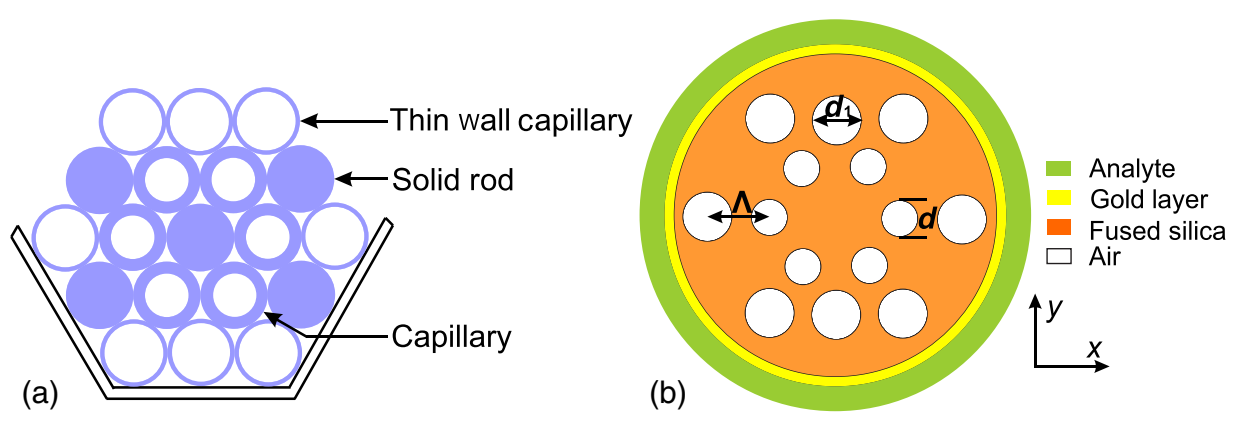

Fig. 1 (a) Cross-section view of the stacked preform of the proposed PCF. (b) Cross-section view of the computational model of the proposed biosensor in the $x y$ plane.

can be created by inserting solid rods. In contrast to others, thick wall capillaries are used for smaller air holes, and the larger air holes can be created using thin wall capillaries. Figure [(b) shows the cross-section of the proposed PCF SPR biosensor, where the PCF consists of a tworing hexagonal lattice design. The center-to-center distance between air holes is $\Lambda=2 \mu \mathrm{m}$, and the core diameter is $1.4 \times \Lambda$. The diameter of air holes in the first and second rings is $d=0.6 \times \Lambda$ and $d_{1}=0.8 \times \Lambda$, respectively. The total size of the PCF is $10 \mu \mathrm{m}$, and the outer diameter of the fused silica [perfectly matched layer (PML) thickness] is $1.5 \mu \mathrm{m}$. The analyte layer thickness is $1.2 \mu \mathrm{m}$. Unlike other PCF sensors, fewer design parameters. The missing central air hole also facilitates the fabrication process. In this design, fused silica was used as the background material.

The RI of fused silica was obtained using the following Sellmeier equation: 2

$$
n^{2}(\lambda)=1+\frac{B_{1} \lambda^{2}}{\lambda^{2}-C_{1}}+\frac{B_{2} \lambda^{2}}{\lambda^{2}-C_{2}}+\frac{B_{3} \lambda^{2}}{\lambda^{2}-C_{3}},
$$

where $n$ is the wavelength-dependent RI of fused silica and $\lambda$ is the wavelength in $\mu \mathrm{m} . B_{1}, B_{2}, B_{3}$, $C_{1}, C_{2}$, and $C_{3}$ are the Sellmeier constants. For fused silica, the constants are 0.69616300, $0.407942600,0.897479400,0.00467914826,0.0135120631$, and 97.9340025 , respectively.

A thin gold layer with a thickness of $t_{\mathrm{g}}=30 \mathrm{~nm}$ is deposited in the outer layer of the PCF. It is a challenging task to maintain a uniform thickness of the outer thin gold layer coated on a circular surface of the PCF. There are several techniques such as radio frequency sputtering, ${ }^{\text {, }}$ thermal evaporation, ${ }^{0}$ and wet-chemistry deposition ${ }^{\mathbb{W}}$ that can be used to deposit this outer metallic layer. However, these metal-coating techniques suffer from extreme surface roughness. In this context, chemical vapour deposition ${ }^{32}$ is an efficient method that permits uniform nanolayer coating with minimal surface roughness. In addition, the newly reported gold atomic layer deposition method, ${ }^{3}$ which can deposit a more uniform gold coating on the curve fiber surface, could be used. The dielectric constant of gold can be obtained from the Drude-Lorentz model ${ }^{\text {B }}$

$$
\varepsilon_{\mathrm{Au}}=\varepsilon_{\infty}-\frac{\omega_{D}^{2}}{\omega\left(\omega+j \gamma_{D}\right)}-\frac{\Delta \varepsilon \Omega_{L}^{2}}{\left(\omega^{2}-\Omega_{L}^{2}\right)+j \Gamma_{L} \omega},
$$

where $\varepsilon_{\mathrm{Au}}$ is the permittivity of gold, $\varepsilon_{\infty}$ is the permittivity at a high frequency with the value of 5.9673, $\omega$ is the angular frequency, which is given by $\omega=2 \pi c / \lambda, c$ is the velocity of light in vacuum, $\omega_{D}$ is the plasma frequency, and $\gamma_{D}$ is the damping frequency. Here, $\omega_{D} / 2 \pi=$ $2113.6 \mathrm{THz}, \gamma_{D} / 2 \pi=15.92 \mathrm{THz}$, and the weighting factor $\Delta \varepsilon=1.09$. The spectral width and oscillator strength of the Lorentz oscillators are given by $\Gamma_{L} / 2 \pi=104.86 \mathrm{THz}$ and $\Omega_{L} / 2 \pi=650.07 \mathrm{THz}$, respectively.

The finite element method-based state-of-the-art COMSOL $v 5.0$ has been used to design and simulate the proposed structure. We imposed circular PML and scattering boundary conditions that absorb outgoing waves from the surface of the PCF. The convergence test was performed to confirm the simulation accuracy. The total computation domain took 30,150 triangular elements to represent the structure. The thickness of the PML and analyte layer was about $14 \%$ and $12 \%$ of the fiber diameter, respectively. 


\section{Results and Discussions}

The fundamental mode field profiles of the core-guided mode and SPP mode for $x$ - and $y$-polarizations are shown in Figs. 2(a) 2(d), respectively. As seen from the figure, in $y$-polarization mode more of the evanescent field reaches the metal layer in comparison with the $x$-polarization mode. As a result, the core-guided mode of $y$-polarization can easily excite the metal electrons to create the SPR effect. Therefore, in the rest of the discussions, we only consider the $y$-polarization mode because of higher loss depth. Although the $y$-polarized mode showed the higher evanescent field compared with the $x$-polarized mode, it is not significant enough to produce a larger amount of propagation loss. More evanescent field interaction with the sample means more propagation loss. Increase of the propagation loss will lead to the higher signal-to-noise ratio; however, if propagation loss is increased, then the sensor length will be reduced to generate the measureable signal. As a result, practical realization will be difficult. For instance, SPR
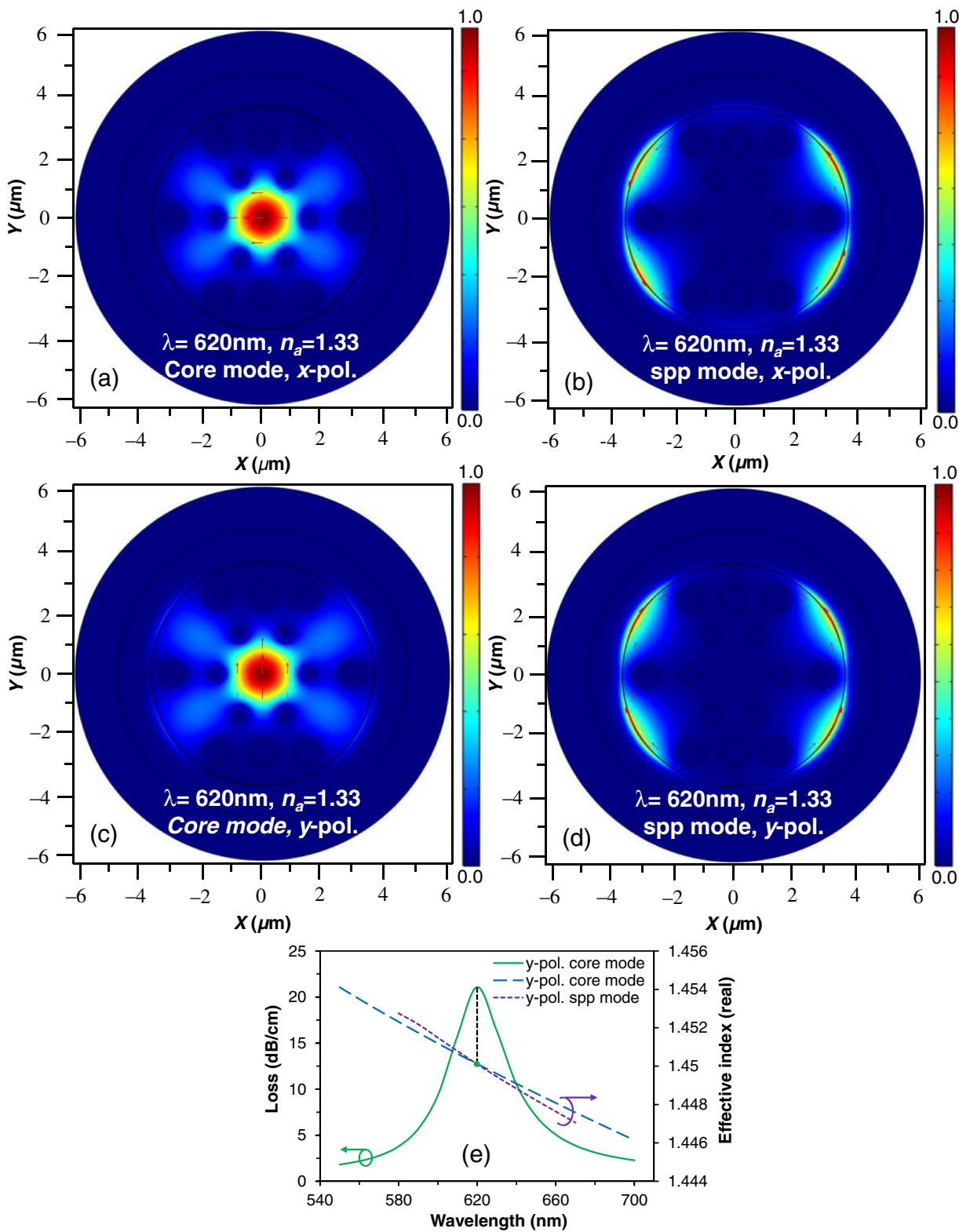

Fig. 2 Mode field distribution of the proposed sensor. (a) and (b) $x$-polarized fundamental coreguided mode and spp mode, respectively; (c) and (d) $y$-polarized fundamental core-guided mode and spp mode, respectively; and (e) dispersion relation between core-guided mode and SPP mode while analyte RI 1.33 . 
sensors are more sensitive compared with other optical sensors, field interaction with the sample is able to detect the unknown sample ${ }^{\text {B. } 55}$ Figure 2(e) represents the relationship between the confinement loss and $n_{\text {eff }}$ of the core mode and the SPP mode for analyte RI $\left(n_{a}\right)$ of 1.33. It is evident that, at a wavelength $(\lambda)$ of $620 \mathrm{~nm}$, the $n_{\text {eff }}$ of the coreguided mode and SPP is equal, which satisfies the required resonance condition. At the resonant wavelength, a sharp loss peak occurs that indicates the maximum power transfer from the coreguided mode to the SPP mode. The unknown analyte can be detected from the resonant wavelength shifts or the amplitude variation of the loss peaks. The confinement loss can be obtained by

$$
\alpha(\mathrm{dB} / \mathrm{cm})=8.686 \times k_{0} \operatorname{Im}\left(n_{\mathrm{eff}}\right) \times 10^{4},
$$

where $\operatorname{Im}\left(n_{\text {eff }}\right)$ is the imaginary part of the effective mode index and $k_{0}$ is the wave number.

A small change of the analyte RI has a significant impact on the loss depth. Loss spectrum of the proposed PCF biosensor with different $n_{a}$ is shown in Fig. 3. It can be observed that increasing $n_{a}$ results in shifting the loss peak toward longer wavelengths (also known as redshift) with an increasing of loss depth. The highest loss depth is $60 \mathrm{~dB} / \mathrm{cm}$ for $n_{a}=1.37$. The higher loss depth indicates stronger power interchange between the core and SPP modes, which results in a narrow resonant spectrum. Linear curve fitting of the resonant wavelength is also shown in the inset of Fig. B. The calculated linearity $R^{2}$ value is 0.9868 , which shows a good linear fitting characteristic. Therefore, the proposed biosensor can be utilized for practical biosensing applications.

The sensitivity of a biosensor can be measured by wavelength interrogation and amplitude interrogation methods. In the wavelength interrogation method, the sensitivity of a biosensor can be calculated from $S_{\lambda}(\lambda)=\Delta \lambda_{\text {peak }} / \Delta n_{a}, \square_{\text {where }} \Delta \lambda_{\text {peak }}$ is the difference between two resonant wavelengths and $\Delta n_{a}$ is the analyte RI difference. Using the wavelength interrogation method, the proposed sensor shows sensitivity of 2000, 3000, 3000, and $4000 \mathrm{~nm} / \mathrm{RIU}$ for $n_{a}$ of 1.33 , $1.34,1.35$, and 1.36 , respectively. When the analyte RI is changed from 1.33 to $1.34,1.34$ to 1.35 , 1.35 to 1.36 , and 1.36 to 1.37 , the calculated $\Delta \lambda_{\text {peak }}$ are $20,30,30$, and $40 \mathrm{~nm}$, respectively. The resolution of the sensor is another important parameter that describes how a small variation of the analyte RI can be detected by the sensor. Resolution $(R)$ of the proposed sensor can be given as

$$
R=\Delta n_{a} \times \Delta \lambda_{\min } / \Delta \lambda_{\text {peak }} \mathrm{RIU},
$$

where $\Delta n_{a}$ is the variation of analyte RI, $\Delta \lambda_{\text {peak }}$ is the minimum spectral resolution, and $\Delta \lambda_{\text {peak }}$ is the maximum resonant wavelength peak shift. Assuming that $\Delta n_{a}=0.01, \Delta \lambda_{\min }=0.1$, $\Delta \lambda_{\text {peak }}=40 \mathrm{~nm}$, and $n_{a}=1.36$, the calculated maximum sensor resolution is $2.5 \times 10^{-5}$ RIU. This resolution is comparable with the results reported in Refs. 12, 20, and 23. Using the amplitude interrogation method, the amplitude sensitivity can be obtained from 1

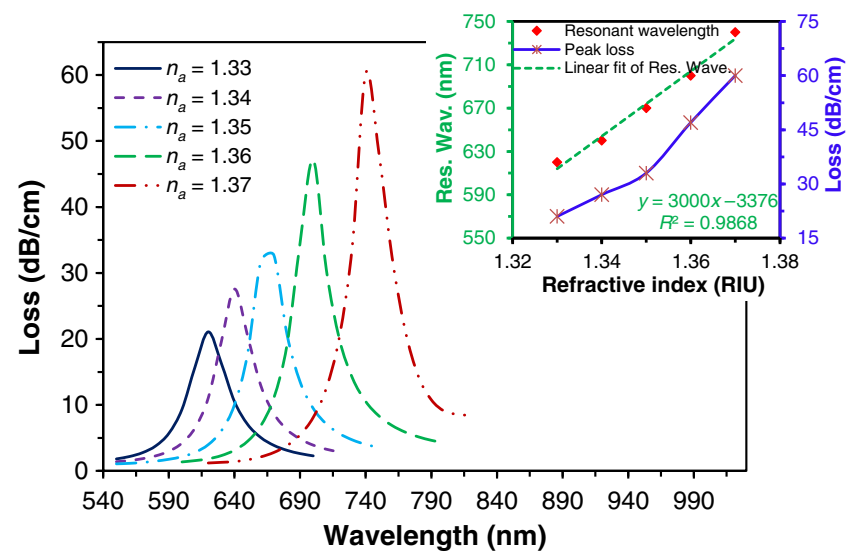

Fig. 3 Loss spectrum of the proposed PCF biosensor for different $n_{a}$ with the variation of wavelength. Linear curve fitting of resonant wavelength is shown in the inset. 


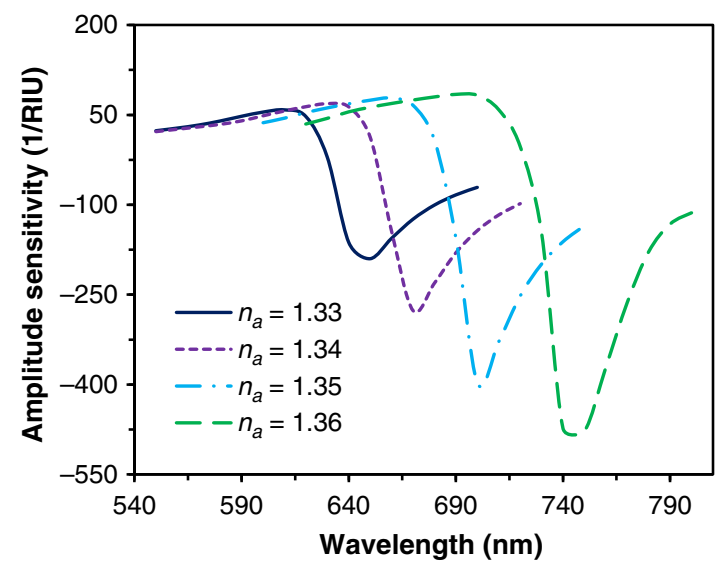

Fig. 4 Amplitude sensitivity of the proposed PCF biosensor as a function of wavelength with different $n_{a}$.

$$
S_{A}\left(\mathrm{RIU}^{-1}\right)=-\frac{1}{\alpha\left(\lambda, n_{a}\right)} \frac{\partial \alpha\left(\lambda, n_{a}\right)}{\partial n_{a}}
$$

where $\alpha\left(\lambda, n_{a}\right)$ is the overall propagation loss at the RI of $n_{a}$ and $\partial \alpha\left(\lambda, n_{a}\right)$ is the loss difference between two loss spectra.

Figure 7 shows the $S_{A}$ as a function of wavelength for different $n_{a}$. As observed from the figure, the proposed sensor shows a maximum amplitude sensitivity of $478 \mathrm{RIU}^{-1}$ when $n_{a}=1.36$. This value is higher than most of the previously reported results tude sensitivities for $n_{a}$ of $1.33,1.34$, and 1.35 are 190,277, and $397 \mathrm{RIU}^{-1}$, respectively. In the case of maximum sensitivity, i.e., $478 \mathrm{RIU}^{-1}$, the calculated sensor resolution is about $2.1 \times 10^{-5}$ RIU taking into consideration that a minimum $1 \%$ of transmitted intensity can be detected accurately.

The thickness of the gold layer has a dominant effect on the performance of the proposed biosensor. The effect of changing $t_{\mathrm{g}}$ for $n_{a}=1.33$ and 1.34 is shown in Fig. 5(a). Due to the increase of $t_{\mathrm{g}}$, loss depth reduces significantly, which is shown in the inset of Fig. 5(a). When $t_{\mathrm{g}}$ is varied from 30 to $50 \mathrm{~nm}$, the corresponding peak loss reduces from 27 to $6 \mathrm{~dB} / \mathrm{cm}$ for $n_{a}=1.34$. This indicates that, with the increase of $t_{\mathrm{g}}$ thickness, the evanescent field interaction with the analyte decreases significantly. The physical reason is the higher damping loss of gold due to the increasing of $t_{\mathrm{g}}$. Moreover, it can be found that increasing $t_{\mathrm{g}}$ causes loss peak shifting toward a longer wavelength. In addition, amplitude sensitivity also shows the same scenario. By increasing the gold thickness from 30 to $50 \mathrm{~nm}$, amplitude sensitivity decreases dramatically, with the values being 190, 155, and $141 \mathrm{RIU}^{-1}$, respectively [shown in Fig. 5(b)]. In addition, the resonant wavelength shifted from 650 to $670 \mathrm{~nm}$ (redshift).
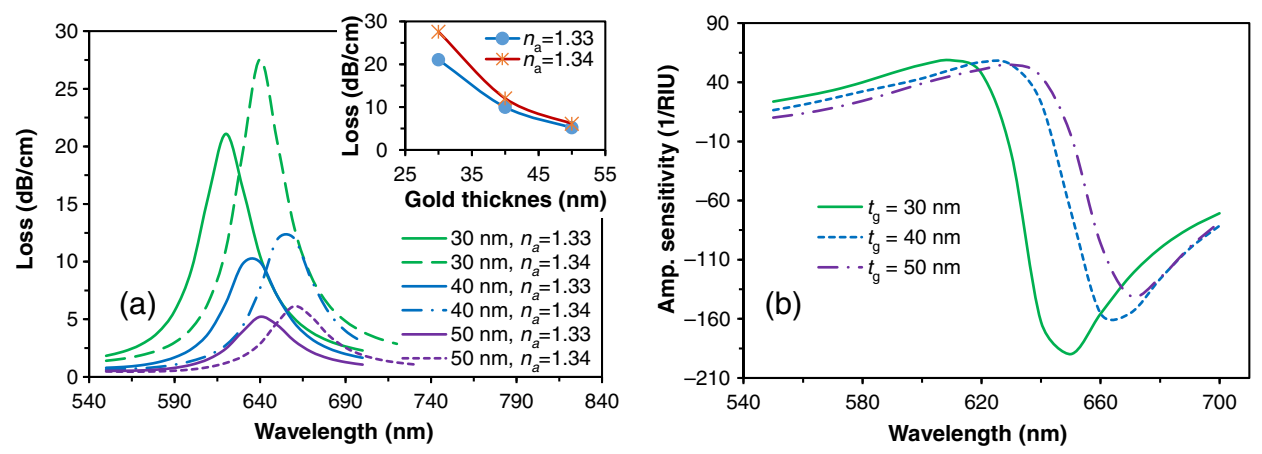

Fig. 5 (a) Loss spectrum of the proposed PCF biosensor for the variation of gold layer thickness with $n_{a}=1.33$ and 1.34. (b) Amplitude sensitivity as a function of wavelength with varying $t_{\mathrm{g}}$ at $n_{a}=1.33$. 

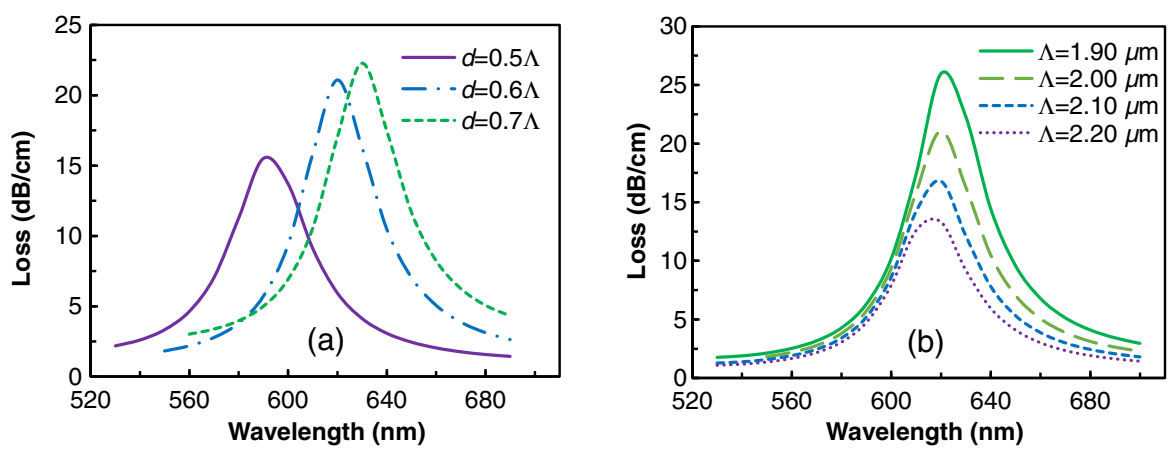

Fig. 6 Loss spectrum of the proposed PCF biosensor for the variation of (a) air-hole dimension and (b) pitch for $n_{a}=1.33$.

The effect of changing the air-hole diameter $d$ on the surface plasmon waves is shown in Fig. 6(a). As $d$ is increased from $0.5 \Lambda$ to $0.7 \Lambda$, the amplitude of peak loss gradually increases with redshifting of the resonant wavelength for a constant $n_{a}$ of 1.33. When $d$ is increased, it results in a decreasing of the index contrast between core and cladding. As a result, peak loss increases, which indicates strong coupling between the guided mode and the SPP mode. In addition, the effect of changing pitch $\Lambda$ on the loss spectrum is shown in Fig. 6(b). Unlike the results obtained from increasing $d$, when the value of $\Lambda$ is increased, the amplitude of the loss peak decreases slowly. Although increasing the value of $d$ shows large redshift, in this case increasing $\Lambda$ results in small blueshift, i.e., changing the resonant peak toward a shorter wavelength. Considering the strength of the coupling between the core and SPP mode, we have selected the optimized values of $d$ and $\Lambda$, which are $0.6 \times \Lambda$ and $2 \mu \mathrm{m}$, respectively.

Table 1 shows the comparison among the properties of the proposed SPR sensor with existing structures in the literature. The performance comparison has taken into account structure type, sensing approach, RI range, wavelength sensitivity, amplitude sensitivity, and sensor resolution. As clearly evident from the table, the proposed SPR PCF sensor shows better performance in terms of amplitude sensitivity, wavelength sensitivity, and sensor resolution.

Table 1 Comparison among the properties of the proposed fiber with previously reported sensors.

\begin{tabular}{|c|c|c|c|c|c|c|}
\hline References & Structure type & $\begin{array}{l}\text { Sensing } \\
\text { approach }\end{array}$ & $\mathrm{RI}$ range & $\begin{array}{l}\text { Wavelength } \\
\text { sensitivity } \\
\text { (nm/RIU) }\end{array}$ & $\begin{array}{l}\text { Amplitude } \\
\text { sensitivity } \\
\left(\mathrm{RIU}^{-1}\right)\end{array}$ & $\begin{array}{l}\text { Resolution } \\
\text { (wavelength } \\
\text { inter.) RIU }\end{array}$ \\
\hline 6 & $\begin{array}{l}\text { Multichannel PCF } \\
\text { sensor }\end{array}$ & $\begin{array}{l}\text { External metal } \\
\text { coating }\end{array}$ & 1.33 to 1.34 & 2400 & - & - \\
\hline 7 & $\begin{array}{l}\text { Selectively ITO-coated } \\
\text { PCF sensor }\end{array}$ & $\begin{array}{l}\text { Internal metal } \\
\text { coating }\end{array}$ & 1.33 to 1.35 & 2000 & 80 & $5 \times 10^{-5}$ \\
\hline 8 & D-shaped PCF sensor & $\begin{array}{l}\text { Internal metal } \\
\text { coating }\end{array}$ & 1.33 to 1.35 & 2520 & 44 & $3.97 \times 10^{-5}$ \\
\hline 13 & Multihole PCF sensor & $\begin{array}{l}\text { Internal metal } \\
\text { coating }\end{array}$ & 1.33 to 1.35 & 2000 & 370 & - \\
\hline 15 & $\begin{array}{l}\text { Silver-graphene-based } \\
\text { PCF sensor }\end{array}$ & $\begin{array}{l}\text { Internal metal } \\
\text { coating }\end{array}$ & 1.46 to 1.49 & 3000 & 418 & $3.33 \times 10^{-5}$ \\
\hline 20 & $\begin{array}{l}\text { Two-ring hexagonal } \\
\text { PCF sensor }\end{array}$ & $\begin{array}{l}\text { External metal } \\
\text { coating }\end{array}$ & 1.33 to 1.37 & 4000 & 320 & $2.5 \times 10^{-5}$ \\
\hline 21 & $\begin{array}{l}\text { Birefringent PCF } \\
\text { sensor }\end{array}$ & $\begin{array}{l}\text { External metal } \\
\text { coating }\end{array}$ & 1.33 to 1.35 & 2000 & 317 & $5 \times 10^{-5}$ \\
\hline This work & $\begin{array}{l}\text { Gold-coated PCF } \\
\text { sensor }\end{array}$ & $\begin{array}{l}\text { External metal } \\
\text { coating }\end{array}$ & 1.33 to 1.37 & 4000 & 478 & $2.5 \times 10^{-5}$ \\
\hline
\end{tabular}


At this stage, we would like to explore the fabrication feasibility of the proposed PCF-based sensor. It is already known that hexagonal lattice PCF can be realized using the most versatile stack-and-draw method 60 (6) As seen in Fig. 1(b), the designed PCF has hexagonal lattice with two air-hole rings. Therefore, the standard stack-and-draw method can be a viable way to realize this PCF in practical form. It should be pointed out that the pitch size is $2 \mu \mathrm{m}$, yielding a total fiber diameter of around $10 \mu \mathrm{m}$. The diameter of the conventional PCF is about $125 \mu \mathrm{m}$. In this case, the proposed PCF could be realized by either stacking the extra solid-rod or two or three times jacketing of the outer layer. Regarding this designed structure, a solid silica surface will be formed in the outside of the cladding (similarly demonstrated in Ref. 38), which can be polished to realize the proposed PCF. Moreover, the proposed PCF can also be realized using a tapered technique .9 In practical sensing applications, generally a white light source/supercontinuum (SC) light source can be used in the visible range to illuminate light into a single mode fiber (SMF) ${ }^{-17}$ A splicing technique or free-space alignment can be used to connect the SMF with the proposed PCF sensor. On the top of the gold surface [according to Fig. [1(b)], a sample analyte channel can be maintained through a pump. The effective RI of the SPP mode will be changed because of the interaction of sample analyte with the ligand; thereby, either blueor redshift will occur. The optical spectrum analyzer (OSA) or photodetector can be used to measure the transmittance light. The output of the OSA or photodetector can be used for further analysis in the computer.

\section{Conclusion}

In summary, we numerically investigated a relatively simple and realistic PCF SPR biosensor based on the SPR effect. Chemically stable gold is used as the plasmonic material as it offers simultaneously a narrow resonance spectrum and large wavelength shift. The proposed biosensor shows a maximum wavelength sensitivity of $4000 \mathrm{~nm} / \mathrm{RIU}$ and a maximum amplitude sensitivity of $478 \mathrm{RIU}^{-1}$ within the sensing range of 1.33 to 1.37 . High sensor resolution of $2.5 \times$ $10^{-5}$ RIU and good linear fitting characteristics are obtained for the optimum design parameters. Due to structural simplicity and excellent sensing properties, the proposed PCF can be regarded as a good biosensor.

\section{References}

1. X. Fan and I. M. White, "Optofluidic microsystems for chemical and biological analysis," Nat. Photonics 5(10), 591-597 (2011).

2. J. Homola, "Present and future of surface plasmon resonance biosensors," Anal. Bioanal. Chem. 377(3), 528-539 (2003).

3. A. Ricciardi et al., "Versatile optical fiber nanoprobes: from plasmonic biosensors to polarization-sensitive devices," ACS Photonics 1(1), 69-78 (2014).

4. A. A. Rifat et al., "Photonic crystal fiber based plasmonic sensors," Sens. Actuators B 243, 311-325 (2017).

5. A. A. Rifat et al., "Highly sensitive multi-core flat fiber surface plasmon resonance refractive index sensor," Opt. Express 24(3), 2485-2495 (2016).

6. S. I. Azzam et al., "Multichannel photonic crystal fiber surface plasmon resonance based sensor," Opt. Quantum Electron. 48(142), 1-11 (2016).

7. J. N. Dash and R. Jha, "SPR biosensor based on polymer PCF coated with conducting metal oxide," IEEE Photonics Technol. Lett. 26(6), 595-598 (2014).

8. X. Yang et al., "Analysis of graphene-based photonic crystal fiber sensor using birefringence and surface plasmon resonance," Plasmonics 12(2), 489-496 (2017).

9. V. Kravets et al., "Graphene-protected copper and silver plasmonics," Sci. Rep. 4, 5517 (2014).

10. M. Schriver et al., "Graphene as a long-term metal oxidation barrier: worse than nothing," ACS Nand 7(7), 5763-5768 (2013).

11. C. Liu et al., "Numerical analysis of a photonic crystal fiber based on a surface plasmon resonance sensor with an annular analyte channel," Opt. Commun. 382, 162-166 (2017). 
12. R. K. Gangwar and V. K. Singh, "Highly sensitive surface plasmon resonance based D-shaped photonic crystal fiber refractive index sensor," Plasmonics 1-6 (2016).

13. D. Gao et al., "Multi-hole fiber based surface plasmon resonance sensor operated at nearinfrared wavelengths," Opt. Commun. 313, 94-98 (2014).

14. W. Qin et al., "Analyte-filled core self-calibration microstructured optical fiber based plasmonic sensor for detecting high refractive index aqueous analyte," Opt. Lasers Eng. 58, 1-8 (2014).

15. A. Rifat et al., "Photonic crystal fiber-based surface plasmon resonance sensor with selective analyte channels and graphene-silver deposited core," Sensors 15(5), 11499-11510 (2015).

16. N. Luan et al., "Surface plasmon resonance sensor based on D-shaped microstructured optical fiber with hollow core," Opt. Express 23(7), 8576-8582 (2015).

17. Q. Xie et al., "Characteristics of D-shaped photonic crystal fiber surface plasmon resonance sensors with different side-polished lengths," Appl. Opt. 56(5), 1550-1555 (2017).

18. M. Tian et al., "All-solid D-shaped photonic fiber sensor based on surface plasmon resonance," Opt. Commun. 285(6), 1550-1554 (2012).

19. A. A. Rifat et al., "Highly sensitive D-shaped photonic crystal fiber based plasmonic biosensor in visible to near-IR," IEEE Sens. J. 17(9), 2776-2783 (2017).

20. A. A. Rifat et al., "Surface plasmon resonance photonic crystal fiber biosensor: a practical sensing approach," IEEE Photonics Technol. Lett. 27(15), 1628-1631 (2015).

21. R. Otupiri et al., "A novel birefrigent photonic crystal fiber surface plasmon resonance biosensor," EEEE Photonics J. 6(4), 6801711 (2014).

22. R. Otupiri, E. Akowuah, and S. Haxha, "Multi-channel SPR biosensor based on PCF for multi-analyte sensing applications," Opt. Express 23(12), 15716-15727 (2015).

23. M. R. Hasan et al., "A highly sensitive gold-coated photonic crystal fiber biosensor based on surface plasmon resonance," Photonics 4(1), 18 (2017).

24. Y. Lu et al., "Surface plasmon resonance sensor based on hollow-core PCFs filled with silver nanowires," Electron. Lett. 51(21), 1675-1677 (2015).

25. Y. Lu et al., "Temperature sensing using photonic crystal fiber filled with silver nanowires and liquid," IEEE Photonics J. 6(3), 6801307 (2014).

26. T. Wieduwilt et al., "Ultrathin niobium nanofilms on fiber optical tapers-a new route towards low-loss hybrid plasmonic modes," Sci. Rep. 5, 17060 (2015).

27. J. O. Trevisanutto, A. Linhananta, and G. Das, "Plasmonic structure: fiber grating formed by gold nanorods on a tapered fiber," Opt. Lett. 41(24), 5789-5792 (2016).

28. W. Sellmeier, "Zur erklärung der abnormen farbenfolge im spectrum einiger substanzen," Ann. Phys. Chem. 219(6), 272-282 (1871).

29. L. Armelao et al., "RF-sputtering of gold on silica surfaces: evolution from clusters to continuous films," Mater. Sci. Eng. Q 25(5-8), 599-603 (2005).

30. M. C. Barnes et al., "Deposition mechanism of gold by thermal evaporation: approach by charged cluster model," J. Cryst. Growth 213(1-2), 83-92 (2000).

31. J. A. Sioss and C. D. Keating, "Batch preparation of linear Au and Ag nanoparticle chains via wet chemistry," Nano Lett. 5(9), 1779-1783 (2005).

32. P. J. A. Sazio, "Microstructured optical fibers as high-pressure microfluidic reactors," Science 311(5767), 1583-1586 (2006).

33. M. B. E. Griffiths et al., "Atomic layer deposition of gold metal," Chem. Mater. 28(1), 44-46 (2016).

34. A. Vial et al., "Improved analytical fit of gold dispersion: application to the modeling of extinction spectra with a finite-difference time-domain method," Phys. Rev. B 71(8), 085416 (2005).

35. Y. Zhong et al., "Review of mid-infrared plasmonic materials," t. Nanophotonics 9(1), 093791 (2015).

36. G. Amouzad Mahdiraji et al., "Challenges and solutions in fabrication of silica-based photonic crystal fibers: an experimental study," Fiber Integr. Opt. 33(1-2), 85-104 (2014).

37. J. Knight et al., "All-silica single-mode optical fiber with photonic crystal cladding," Opt. Lett. 21(19), 1547-1549 (1996).

38. Y. S. Chen et al., "Harmonic mode-locked fiber laser based on photonic crystal fiber filled with topological insulator solution," Photonics 2(2), 342-354 (2015). 
39. J. K. Chandalia et al., "Adiabatic coupling in tapered air-silica microstructured optical fiber," IEEE Photonics Technol. Lett. 13(1), 52-54 (2001).

40. K. Bremer and B. Roth, "Fibre optic surface plasmon resonance sensor system designed for smartphones," Opt. Express 23(13), 17179-17184 (2015).

41. Y. Zhao, Z. Q. Deng, and Q. Wang, "Fiber optic SPR sensor for liquid concentration measurement," Sens. Actuators B 192, 229-233 (2014).

42. H. H. Jeong et al., "Fabrication of fiber-optic localized surface plasmon resonance sensor and its application to detect antibody-antigen reaction of interferon-gamma," Opt. Eng. 50(12), 124405 (2011).

Ahmmed A. Rifat is a PhD student at Australian National University, Canberra, Australia. $\mathrm{He}$ received his MEngSc degree in electrical engineering from the University of Malaya, Malaysia, in January 2016. He also worked as a research assistant at the Integrated Lightwave Research Group, University of Malaya, Kuala Lumpur, Malaysia. His research interests include surface plasmon resonance, dielectric metasurfaces, photonic crystal fiber, optical sensors, and optical devices. He has published over 20 peer-reviewed journal articles.

Md. Rabiul Hasan received his MSc engineering degree in electrical and electronic engineering from Rajshahi University of Engineering and Technology (RUET), Rajshahi, Bangladesh, in 2016. He received his BSc Eng degree in electronics and telecommunication engineering (ETE) from RUET, in 2012. Currently, he is working as an assistant professor in the ETE Department, RUET. His current research interests include photonic crystal fibers, terahertz wave propagation, and surface plasmon resonance biosensor.

Rajib Ahmed is a PhD student at the School of Engineering, University of Birmingham, United Kingdom. His research focuses on photonic devices based on nanostructures, including laserbased nanofabrication, carbon nanotubes (CNTs), waveguide, microring resonator, photonic crystals, and graphene-based photonic devices modeling and implementation.

Haider Butt is a lecturer (assistant professor) at the School of Engineering, University of Birmingham, United Kingdom. Previously, he was a Henslow research fellow at the University of Cambridge, United Kingdom, from where he received his PhD in 2012. His research focuses on photonic devices based on nanostructures such as CNT, graphene, and plasmonic nanostructures. He has published over 50 peer-reviewed journal articles and has secured several prestigious research awards. 